\title{
Phenylethyl isothiocyanate reverses cisplatin resistance in biliary tract cancer cells via glutathionylation-dependent degradation of $\mathrm{Mcl}-1$
}

\author{
Qiwei Li ${ }^{1}$, Ming Zhan ${ }^{1}$, Wei Chen ${ }^{1}$, Benpeng Zhao ${ }^{2}$, Kai Yang ${ }^{2}$, Jie Yang ${ }^{2}$, Jing $\mathbf{Y i}^{2}$, \\ Qihong Huang ${ }^{3}$, Man Mohan'2, Zhaoyuan Hou ${ }^{2}$, Jian Wang ${ }^{1}$ \\ ${ }^{1}$ Department of Biliary-Pancreatic Surgery, Ren Ji Hospital, School of Medicine, Shanghai Jiao Tong University, Shanghai, China \\ ${ }^{2}$ Department of Biochemistry and Molecular Cell Biology, Shanghai Key Laboratory of Tumor Microenvironment and \\ Inflammation, Institutes of Medical Sciences, Shanghai Jiao Tong University, School of Medicine, Shanghai, China \\ ${ }^{3}$ The Wistar Institute, University of Pennsylvania and Veterans Affairs Medical Center, Philadelphia, Pennsylvania, USA \\ Correspondence to: Jian Wang, e-mail: dr_wangjian@126.com
}

Keywords: biliary tract cancer, PEITC, cisplatin, Mcl-1, glutathionylation

Received: July 06, 2015

Accepted: January 21, 2016

Published: February 03, 2016

\section{ABSTRACT}

Biliary tract cancer (BTC) is a highly malignant cancer. BTC exhibits a low response rate to cisplatin (CDDP) treatment, and therefore, an understanding of the mechanism of CDDP resistance is urgently needed. Here, we show that BTC cells develop CDDP resistance due, in part, to upregulation of myeloid cell leukemia 1 (Mcl-1). Phenylethyl isothiocyanate (PEITC), a natural compound found in watercress, could enhance the efficacy of CDDP by degrading Mcl-1. PEITC-CDDP co-treatment also increased the rate of apoptosis of cancer stem-like side population (SP) cells and inhibited xenograft tumor growth without obvious toxic effects. In vitro, PEITC decreased reduced glutathione (GSH), which resulted in decreased GSH/oxidized glutathione (GSSG) ratio and increased glutathionylation of $\mathrm{Mcl}-1$, leading to rapid proteasomal degradation of Mcl-1. Furthermore, we identified Cys16 and Cys286 as Mcl-1 glutathionylation sites, and mutating them resulted in PEITC-mediated degradation resistant $\mathrm{Mcl}-1$ protein. In conclusion, we demonstrate for the first time that CDDP resistance is partially associated with $\mathrm{Mcl-1}$ in BTC cells and we identify a novel mechanism that PEITC can enhance CDDP-induced apoptosis via glutathionylation-dependent degradation of $\mathrm{Mcl}-1$. Hence, our results provide support that dietary intake of watercress may help reverse CDDP resistance in BTC patients.

\section{INTRODUCTION}

Biliary tract cancer (BTC) refers to a group of cancers of the biliary tract, including gallbladder cancer, cholangiocarcinoma of intrahepatic and extrahepatic bile ducts, and cancers of the ampulla and papilla of Vater [1]. BTC is a common form of cancer in East Asia and Latin America [2,3] and surgical resection is the only curative treatment. However, most patients are diagnosed with advanced-stage disease, making them ineligible for complete surgical resection. The prognosis for patients with advanced BTC is very poor, and most survive for less than a year after diagnosis. Cisplatin (CDDP) based chemotherapy is widely used to treat patients with advanced BTC [4]. However, BTC cells are highly chemoresistant. The mechanism of CDDP resistance in BTC cells is poorly understood. Therefore, investigation into the mechanism of CDDP resistance and strategies to alleviate the CDDP resistance are urgently needed.

Various naturally occurring compounds are being tested for their anti-tumor activity. Such compounds when used in combination with known chemotherapeutic agents may help in overcoming chemoresistance, and may provide new strategies and ideas for treatment in clinic. Phenylethyl isothiocyanate (PEITC) is present in high concentrations as its precursor gluconasturtiin in cruciferous vegetables, such as watercress. Upon chewing or chopping, PEITC is released as a product of hydrolysis mediated by myrosinase [5]. Accumulating evidence indicates that PEITC can inhibit cell growth and 
induce apoptosis in a variety of cancer cells, suggesting its potential value as an anticancer agent or an adjunctive therapy to current cancer treatment strategies [6-8]. Additionally, it was shown that eating watercress can significantly increase the blood level of PEITC in humans [9]. These studies and their results gave us important clue to study the effect of combined treatment with PEITC and CDDP on BTC cells.

Though recent data has shown that PEITC can sensitize some tumor cells to CDDP $[5,10-12]$, the synergistic effect of PEITC and CDDP in BTC cells has not been investigated. Here, we demonstrate for the first time that CDDP resistance is partially associated with Mcl-1 in BTC cells, and PEITC can enhance CDDP-induced apoptosis via glutathionylation-dependent degradation of myeloid cell leukemia 1 (Mcl-1). Interestingly and promisingly, PEITC-CDDP co-treatment can increase the rate of apoptosis of cancer stem-like side population (SP) cells and significantly reduce the growth of xenograft tumor without any major toxic effects. Our results suggests that dietary intake of watercress, which is a rich source of PEITC, may help reverse CDDP resistance in BTC patients.

\section{RESULTS}

\section{PEITC enhances CDDP-induced inhibition of cell viability in BTC cells by increasing apoptosis}

To examine the effect of PEITC-CDDP co-treatment on cell viability, human gallbladder cancer GBC-SD cells were treated with PEITC, CDDP, or a PEITC-CDDP combination, and cell metabolic activity was measured using the 3-(4, 5-dimethylthia-zol-2-yl)-2, 5-diphenyltetrazolium bromide (MTT) assay. Notably, PEITC-CDDP co-treatment led to a significant reduction in cell viability (Figure 1A). To quantify synergism, the median-drug effect analysis method was used and the Combination Index (CI) values were calculated. Synergism is indicated by a CI of less than 1, additivity by a CI equal to 1 , and antagonism by a CI greater than 1 . Normalised isobolograms (Figure 1B) display data points below the additivity line, indicating synergy in growth inhibition of GBC-SD cells. Drug Reduction Index (DRI) was then calculated (Table 1). These results suggest that PEITCCDDP co-treatment possesses a synergistic effect on GBC-SD cell proliferation.

To determine whether PEITC in combination with CDDP decreases cell viability via an increase in apoptosis, annexin V-fluorescein isothiocyarate (Annexin V-FITC)/propidium iodide (PI) double labeling flow cytometry was used to determine the percentage of cells entering apoptosis. Flow cytometry analysis showed that CDDP caused about $10 \%$ of the cells to enter apoptosis, but co-treatment with PEITC dramatically enhanced CDDP-induced apoptosis to $40 \%$ (Figure 1C-1D).
A similar pro-apoptotic effect of the combined treatment with PEITC and CDDP was also observed in human cholangiocarcinoma RBE cells (Figure 1E-1H). Together, these data demonstrated that PEITC can enhance CDDPinduced apoptosis in BTC cells.

\section{PEITC enhances the sensitivity of SP cells and xenograft tumors to CDDP}

Recent studies have shown that SP cells isolated from various cancer cell lines and primary tumors possess cancer stem-like properties [13-16]. SP cells can effectively avoid the effects of chemotherapeutic drugs, and are considered to be the root cause of tumor recurrence and metastasis. Therefore, we tested the effect of PEITCCDDP co-treatment on SP cells from GBC-SD cells. The proportions of SP cells was 5.2\% (Figure 2A). As shown in Figure 2B, flow cytometry analysis of SP cells treated with PEITC, CDDP, or a PEITC-CDDP combination showed that CDDP alone caused little cell apoptosis, but when combined with PEITC, markedly enhanced apoptosis at $24 \mathrm{hrs}$. These results demonstrate that PEITC significantly enhances the sensitivity of SP cells to CDDP.

To further examine the synergistic effect of PEITC and CDDP in vivo, GBC-SD cells were transplanted into nude mice. When the tumor size reached approximately $50 \mathrm{~mm}^{3}$, mice were randomly sorted into four equal groups. The tumor-bearing mice were intra-peritoneally injected with physiological saline as a control, PEITC, CDDP or PEITC-CDDP combination for 10 days. Treatment of mice with CDDP alone moderately inhibited tumor growth, but PEITC-CDDP combination treatment resulted in a striking reduction in the average tumor weight by about $50 \%$ (Figure $2 \mathrm{C}$ ). The potent in vivo anticancer effect in the PEITC-CDDP combined group was further evident in the tumor growth curve data (Figure 2D). Systemic toxic effects of the treatments in these mice were evaluated by measuring the loss in body weight. No notable differences were observed between the treated groups (Figure 2E). Collectively, these results demonstrate that PEITC-CDDP co-treatment can effectively inhibit tumor growth without obvious toxic effects in vivo.

\section{Mcl-1 is partially responsible for CDDP resistance in GBC-SD and SP cells}

Anti-apoptotic proteins such as B-cell lymphoma 2 (Bcl-2), B-cell lymphoma-extra large (Bcl-xl) and Mcl-1 are known to play key roles in cancer cell apoptosis. Therefore, we investigated the relationship between these anti-apoptotic proteins and CDDP resistance in GBC-SD cells. Interestingly, CDDP treatment increased Mcl-1 protein level in a time- and dose-dependent manner, but did not increase Bcl-2 or Bcl-xl protein amounts (Figure 3A). To determine if Mcl-1 is playing a role in the 
cytotoxic sensitivity of GBC-SD cells to CDDP, cells were transfected with two siRNA oligonucleotides targeting Mcl-1 (Figure 3B), followed by CDDP treatment for $24 \mathrm{hrs}$. Knock down of Mcl-1 increased CDDP-induced apoptosis (Figure 3C). We also observed that Mcl-1 protein level was higher in SP cells than that in main population (MP) cells (Figure 3D). These data suggest that Mcl-1 is partially responsible for CDDP resistance in GBC-SD and SP cells.

\section{PEITC enhances the cytotoxicity of CDDP through proteasomal degradation of Mcl-1 in vitro and in vivo}

To better understand the mechanism of how PEITC overcomes CDDP resistance, we analyzed Mcl-1 protein level in lysates from GBC-SD cells treated with PEITC and/or CDDP. PEITC treatment decreased Mcl-1 expression in a time- and dose-dependent manner (Figure 4A-4B). Notably, combined treatment with PEITC and CDDP significantly decreased Mcl-1 protein level compared to CDDP alone (Figure 4C). In SP cells, the PEITC-CDDP treatment also lead to a significant decrease in Mcl-1 protein level compared to treatment only with CDDP (Figure 4D). To investigate the effect of PEITC-CDDP treatment on Mcl-1 protein level in vivo, tumor tissues harvested from two mice of each group were examined by immunoblotting. PEITC and/or CDDPmediated changes in Mcl-1 protein level in the tumor tissue were generally in agreement with the molecular alteration observed in cultured cells (Figure 4E). Furthermore, exogenous overexpression of Mcl-1 impeded PEITC-CDDP-induced apoptosis (Figure 4F-4G). These data suggest that PEITC enhances the cytotoxicity of CDDP through a reduction in $\mathrm{Mcl}-1$ in vitro and in vivo.
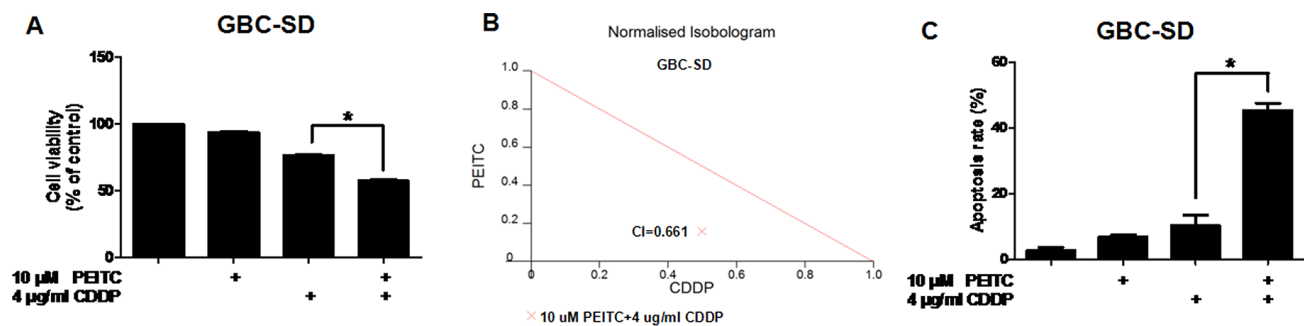

D

GBC-SD
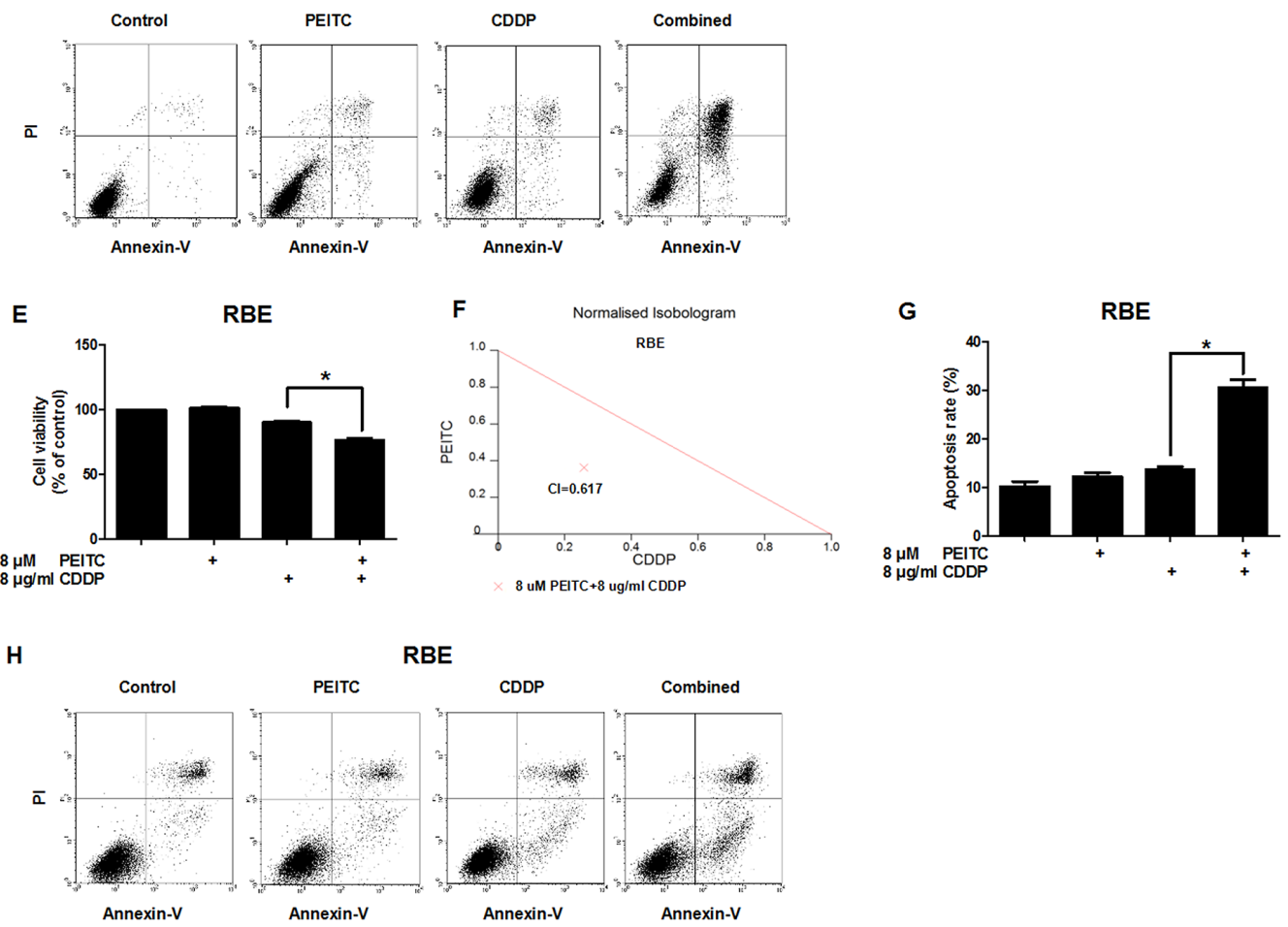

RBE

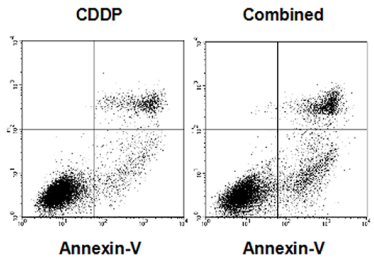

Figure 1: PEITC enhances CDDP-induced apoptosis in GBC-SD and RBE cells. (A) Cells were treated with PEITC, CDDP or PEITC-CDDP combination for $24 \mathrm{hrs}$ and prepared for MTT assays. (B) CI of PEITC-CDDP treatment in GBC-SD cells. (C) Flow cytometry analysis of rate of apoptosis in GBC-SD cells (Annexin V/PI flow cytometry, bar charts). (D) Apoptosis in GBC-SD cells (density plots). (E) Cell viability assay of RBE cells (MTT). (F) CI in RBE cells. (G) Flow cytometry analysis of rate of apoptosis in RBE cells (Annexin V/PI flow cytometry, bar charts). (H) Apoptosis in RBE cells (density plots). Data shown is average of three independent experiments. $* P<0.05$. 
Table 1: CI and DRI values of PEITC and CDDP in BTC cells

\begin{tabular}{|c|c|c|c|c|c|c|}
\hline Cell line & $\begin{array}{c}\text { PEITC } \\
\text { (uM) }\end{array}$ & $\begin{array}{c}\text { CDDP } \\
\text { (ug/ml) }\end{array}$ & $\mathbf{F a}^{\mathbf{a}}$ & $\mathbf{C I}^{\mathrm{b}}$ & $\begin{array}{c}\text { DRI }^{\mathbf{c}} \\
\text { PEITC }\end{array}$ & $\begin{array}{c}\text { DRI } \\
\text { CDDP }\end{array}$ \\
\hline GBC-SD & 10 & 4 & 0.4282 & 0.661 & 6.157 & 2.006 \\
\hline RBE & 8 & 8 & 0.2329 & 0.617 & 2.764 & 3.924 \\
\hline
\end{tabular}

a. Fa: fractional inhibition.

b. $\mathrm{CI}<1,=1$, and $>1$ indicates synergism, additive effect, and antagonism, respectively.

c. DRI: fold of dose reduction for each drug in combination, for a given degree of inhibition, when compared with the dose of each drug alone for the same degree of inhibition. A DRI greater than 1 indicates an enhanced cytotoxicity for the combination.

To determine if the decrease in Mcl-1 protein level caused by PEITC was due to transcriptional inhibition or post-transcriptional regulation, we examined $\mathrm{Mcl}-1 \mathrm{mRNA}$ level by quantitative real time PCR in GBC-SD cells treated with PEITC. Surprisingly, PEITC increased Mcl-1 mRNA level (Figure 4H). This suggested that
PEITC-mediated decrease of Mcl-1 expression is regulated post-transcriptionally. Western blot analysis showed that Mcl-1 degradation was facilitated after 6 hours of PEITC treatment (Figure 4I). Next, to ask if PEITC mediated degradation of Mcl-1 involves proteasomal degradation, we treated GBC-SD cells with the proteasome inhibitor
A

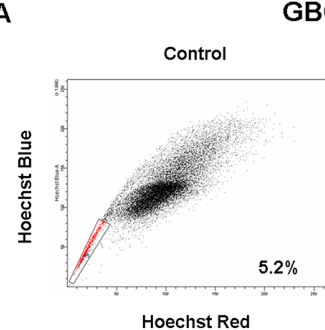

GBC-SD

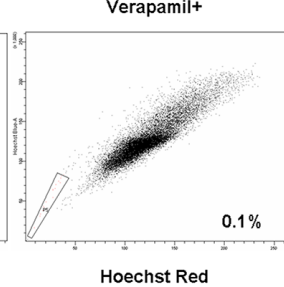

C

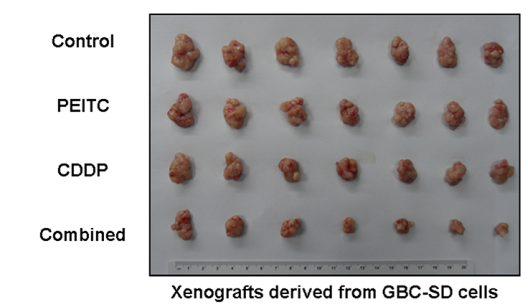

D

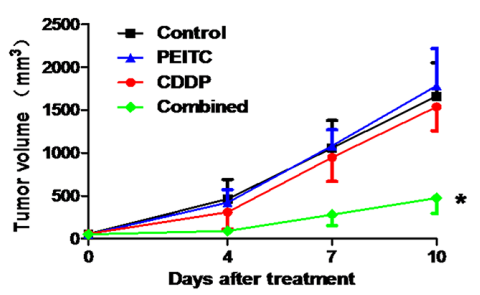

B

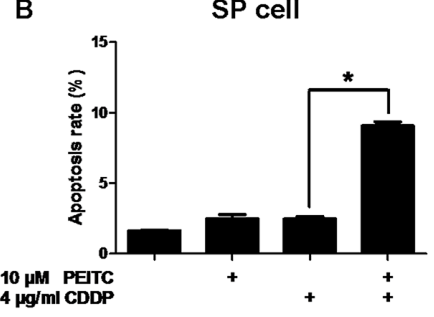

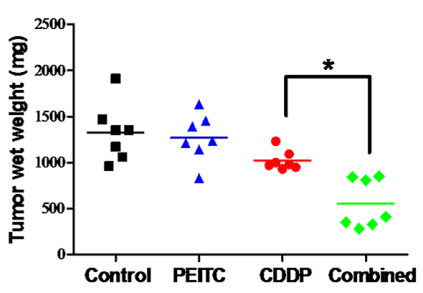

E

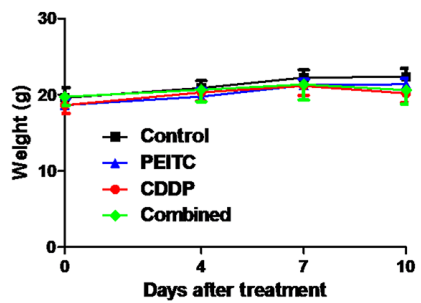

Figure 2: PEITC-CDDP co-treatment sensitizes SP cells and inhibits xenograft tumor growth without obvious toxic effects. (A) FACS analysis on single cell suspension of GBC-SD cells stained with Hoechst 33342 dye showing SP cells. SP cells are enclosed within the area demarcated in black. Verapamil inhibited the efflux of the dye and caused the disappearance of SP cells. A representative plot of the frequency of SP cells is provided. (B) SP cells from GBC-SD cells were treated with PEITC, CDDP or PEITCCDDP combination for $24 \mathrm{hrs}$ and apoptosis detected by Annexin V/PI assay. Data shown is average of three independent experiments. $* P<0.05$. (C) GBC-SD cells were transplanted into nude mice. When tumor size reached approximately $50 \mathrm{~mm}^{3}$, mice were randomly sorted into four equal groups. The tumor-bearing mice were intra-peritoneally injected with physiological saline as a control, PEITC, CDDP or PEITC-CDDP combination for 10 days. Xenografts were excised and weighed. Each dot represents weight of one tumor, and the mean tumor weights of each group is indicated by solid lines (right panel; $n=7$ ). ${ }^{*} P<0.05$. (D) Volume of the tumors was measured twice a week, and a tumor growth curve created for each group $(n=7)$. ${ }^{*} P<0.05$,. (E) Mice were weighed twice a week, and a weight curve created for each group $(n=7)$. 
MG132 and found that the treatment recovered Mcl-1 protein amount to normal level (Figure 4J). Taken together, these data indicate that PEITC decreases Mcl-1 protein level via proteasomal degradation.

\section{PEITC induces proteasomal degradation of Mcl-1 through depletion of reduced glutathione (GSH) and decrease of GSH/oxidized glutathione (GSSG) ratio}

Previous studies have shown that PEITC can alter the redox state of cancer cells through GSH reduction $[6,7,17]$. Since Mcl-1 is a redox sensitive protein [7], we analyzed the relationship between GSH reduction and Mcl-1 degradation in GBC-SD cells. Analysis of GSH revealed that PEITC induced a rapid GSH depletion, detectable after 1 hour of treatment (Figure 5A). As shown in Figure 5B-5C, PEITC increased GSSG levels and decreased GSH/GSSG ratio, which reflects the cellular redox state, after 6 hours of treatment. In comparison, CDDP only induced GSH and GSSG reduction with no apparent reduction in GSH/GSSG ratio (Figure 5D-5F). Therefore, these data suggest that PEITC can induce oxidative stress in GBC-SD cells. Coincidently, PEITC also facilitated Mcl-1 degradation after 6 hours of treatment (Figure 4B). Since there was no Mcl-1 degradation in the first few hours of PEITC incubation, it is likely that the depletion of GSH was a primary event that triggered a decrease in $\mathrm{GSH} /$ GSSG ratio and subsequent Mcl-1 degradation. In support of this hypothesis, supplementing cell culture medium with GSH precursor N-acetylcysteine (NAC) prevented
PEITC-induced GSH depletion (Figure 5G), a decrease in $\mathrm{GSH} / \mathrm{GSSG}$ ratio (Figure $5 \mathrm{H}$ ), and Mcl-1 degradation (Figure 5I). Also, it significantly suppressed PEITC-CDDPinduced cell apoptosis (Figure 5J). Taken together, these data suggest that PEITC induces proteasomal degradation of Mcl-1 through depletion of GSH and a decrease in GSH/ GSSG ratio.

\section{PEITC induces proteasomal degradation of Mcl-1 by increasing the glutathionylated Mcl-1}

Since Mcl-1 is a target of glutathionylation [7] and protein glutathionylation is greatly enhanced by decreased GSH/GSSG ratios that accompany cellular oxidative stress [18], we speculated that PEITC could increase the glutathionylated Mcl-1. Firstly, we found that endogenous Mcl-1 was partially glutathionylated under non-stressed conditions (Figure 6A). Furthermore, we found that DL-Dithiothreitol (DTT), a reducing agent, decreased the glutathionylated Mcl-1, and PEITC increased the glutathionylated Mcl-1 in a time-dependent manner (Figure 6B-6C).

Previous studies have demonstrated that glutathionylation of certain proteins may affect their functions and stability $[18,19]$. We speculated that the glutathionylated Mcl-1 may be more susceptible to proteasomal degradation. Since only two cysteine residues, Cys16 and Cys 286, exist in the Mcl-1 protein, we investigated both these sites for potential glutathionylation. We used site-directed mutagenesis to convert these two cysteine residues Cys16 and Cys286, to serines (C16S, C286S, and C16S/C286S).
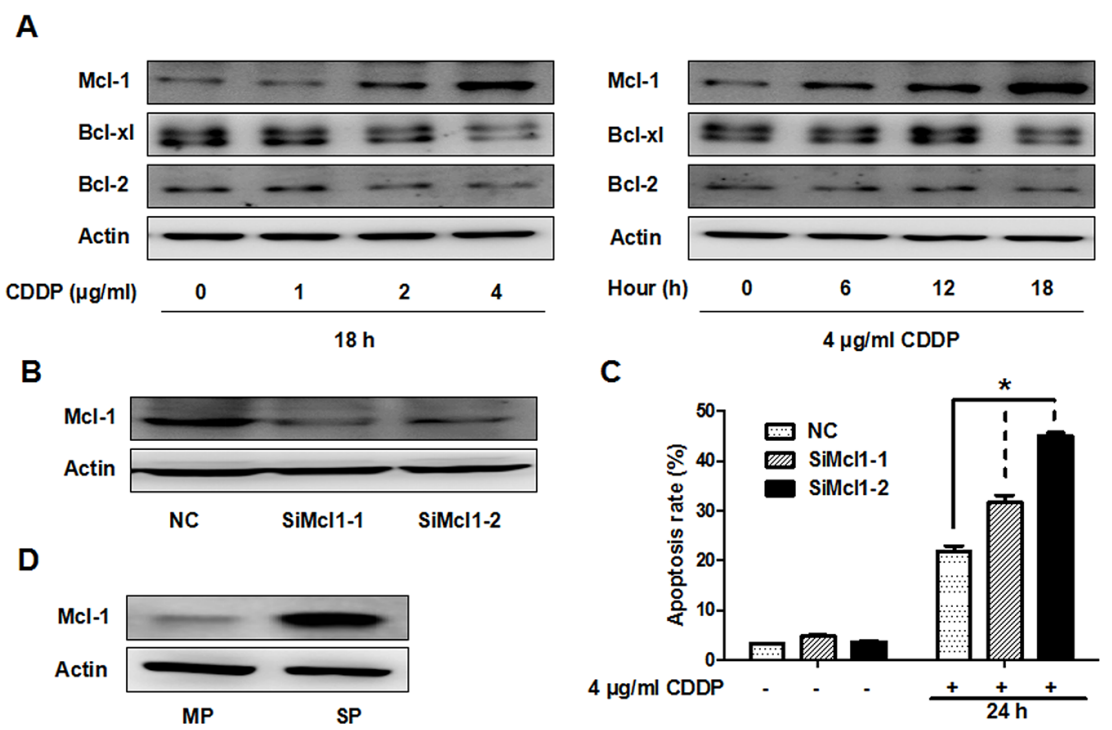

Figure 3: CDDP resistance is partially associated with Mcl-1 in GBC-SD and SP cells. (A) Immunoblot analysis of Mcl-1, Bcl-xl and Bcl-2 protein in GBC-SD cells. Left panel, cells were treated with the indicated concentrations of CDDP for 18 hrs. Right panel, cells were treated with $4 \mathrm{ug} / \mathrm{ml} \mathrm{CDDP}$ and harvested at the indicated times. B-Actin was used as a loading control. (B) Cells were transfected with non-specific siRNA (NC) or Mcl-1 siRNA (SiMcl1) for $48 \mathrm{hrs}$ and reduction in Mcl-1 was analysed by western blot (C) Apoptosis analysis using Annexin V/PI flow cytometry in GBC-SD cells transfected with $M c l-1$ siRNA after treatment with CDDP for 24 hrs. Data shown is average of three independent experiments. ${ }^{*} P<0.05$. (D) Immunoblot analysis of Mcl-1 protein level in SP and MP cells from GBC-SD cells. 
By examining the glutathionylation of Flag-Mcl-1 wild type (WT) and mutants, we found that the C16S mutant was weakly glutathionylated and the C286S mutant was modestly glutathionylated (Figure 6D, lanes 3-4), whereas the double mutant was devoid of any glutathionylation (Figure 6D, lane 5). Taken together, these findings suggest that both the two cysteine residues of Mcl-1 are glutathionylation

A

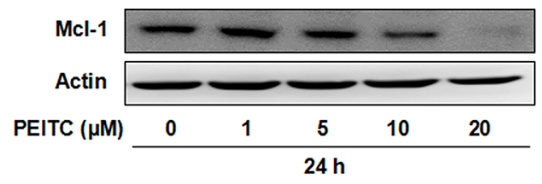

C

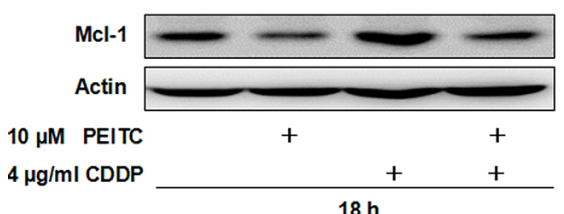

E

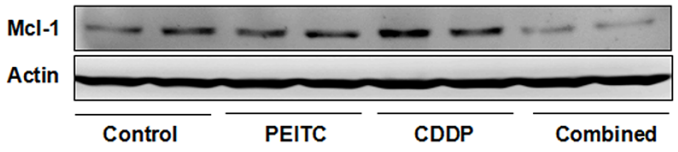

G

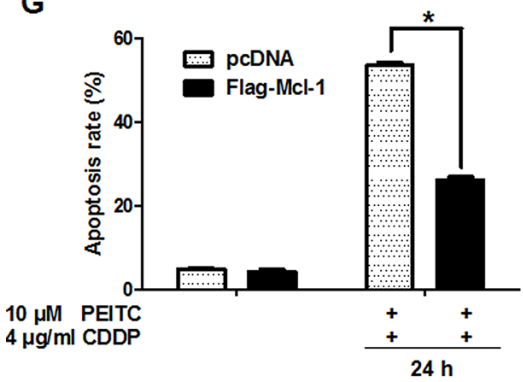

I

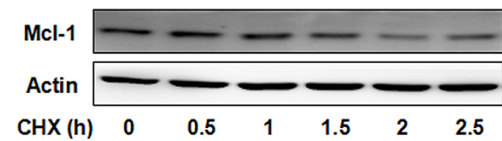

Mcl-1

Actin

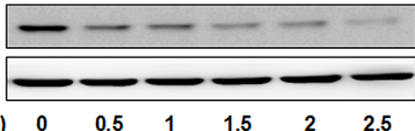

sites. Finally, cells expressing Flag-Mcl-1 WT and C16S/ C286S mutant were treated with PEITC for $24 \mathrm{hrs}$, and their protein levels were determined. Flag-Mcl-1 WT protein was decreased in PEITC-treated cells, while the C16S/C286S mutant was not sensitive to PEITC-mediated degradation (Figure 6E), suggesting that PEITC induces glutathionylation-dependent degradation of Mcl-1.

B

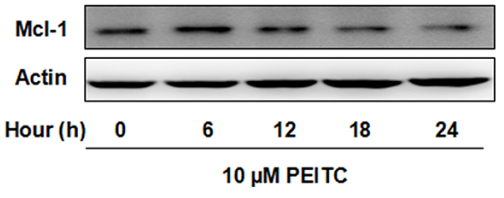

D

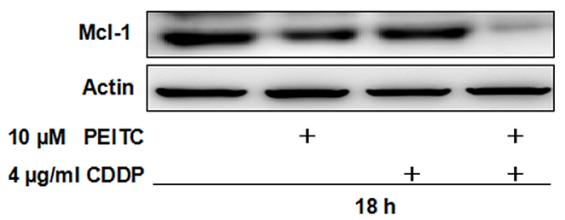

F

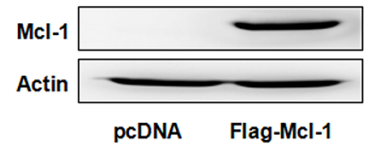

H

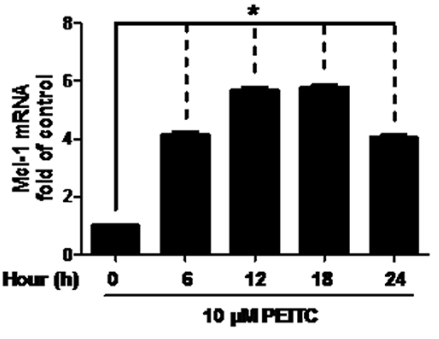

J

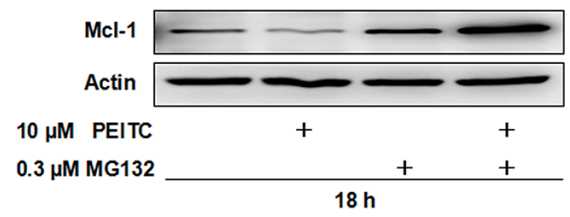

Figure 4: PEITC induces proteasomal degradation of Mcl-1 in vitro and in vivo. (A) Immunoblot analysis of Mcl-1 in GBC-SD cells after treatment with the indicated concentrations of PEITC for 24 hrs. (B) Mcl-1 protein was analysed after treatement with $10 \mathrm{uM}$ PEITC for various times $(\mathbf{C}) \mathrm{Mcl}-1$ protein level was analysed after treatment with PEITC, CDDP or PEITC-CDDP combination for $18 \mathrm{hrs}$. (D) SP cells from GBC-SD cells were treated with PEITC, CDDP or PEITC-CDDP combination for $18 \mathrm{hrs,} \mathrm{and} \mathrm{Mcl-1} \mathrm{protein}$ level analyzed. (E) Immunoblot analysis of Mcl-1 protein in tumor tissue extracts from control group; PEITC group; CDDP group; and PEITC-CDDP combination group. Tumor tissues were from two mice of each group. (F) Mcl-1 protein level was determined by western blot after transfection with vector or $\mathrm{Mcl}-1$ plasmid for $48 \mathrm{hrs}$. (G) Apoptosis analysis using Annexin V/PI flow cytometry in GBC-SD cells transfected with $M c l-1$ plasmid after treatment with PEITC-CDDP combination for 24 hrs. (H) Time course analysis of $M c l-1 \mathrm{mRNA}$ by $10 \mathrm{uM}$ PEITC in GBC-SD cells, detected by quantitative real time PCR analysis. Data shown is average of three independent experiments. $* P<0.05$. (I) Immunoblot analysis of Mcl-1 protein in GBC-SD cells. Cells were treated with $20 \mathrm{ug} / \mathrm{ml}$ cycloheximide for the indicated times or were pretreated with $10 \mathrm{uM}$ PEITC for $6 \mathrm{hrs}$ before exposure to $20 \mathrm{ug} / \mathrm{ml}$ cycloheximide. (J) Mcl-1 protein was analysed in cells treated with PEITC, MG132 or PEITC-MG132 combination for 18 hrs. ß-Actin was used as a loading control. CHX: cycloheximide. 


\section{DISCUSSION}

CDDP-based chemotherapy is an important treatment regimen used in the clinical management of BTC, but the mechanisms for CDDP resistance are not entirely clear. Cancer cells may become resistant to platinum-based drugs through multiple mechanisms, such as an increased ability to repair DNA damage caused by platinum, neutralization of platinum toxicity, blocking platinum entry into the nucleus, an increase in drug export and so on [11, 20-24]. In addition, cisplatin resistance can develop through an increased ability to avoid druginduced cell damage, cell shrinkage and hence initiation of apoptosis [25]. Apoptosis is regulated in part by the Bcl-2 family of proteins which consists of both proapoptotic and anti-apoptotic proteins [26]. Among all the anti-apoptotic Bcl-2 family members, Mcl-1 functions as a major survival factor, particularly in solid cancers [27].
A

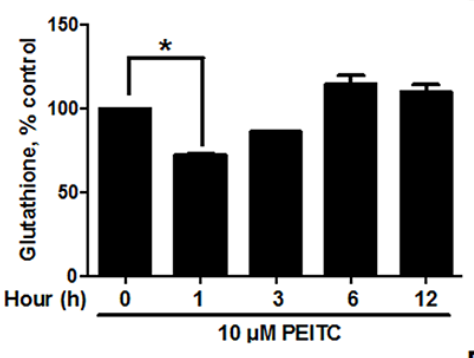

D

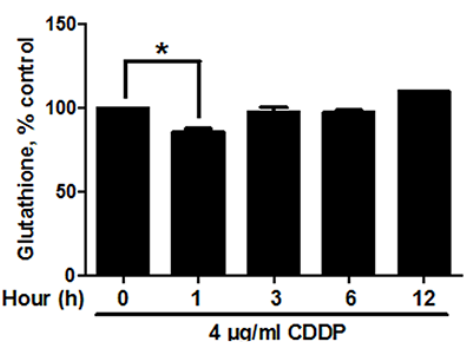

G

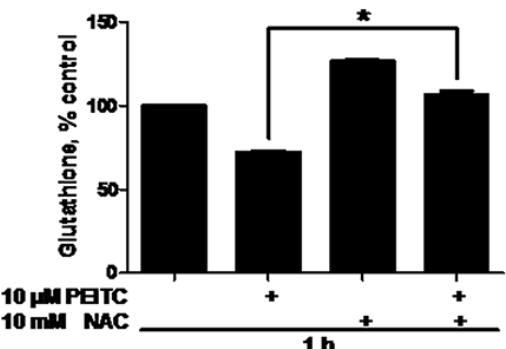

I

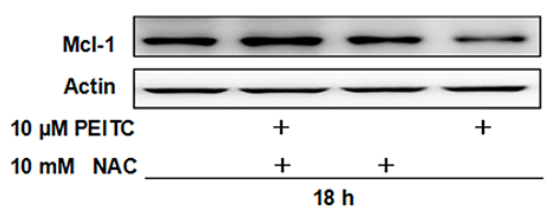

B

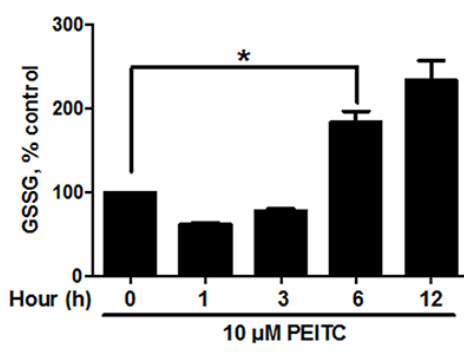

E

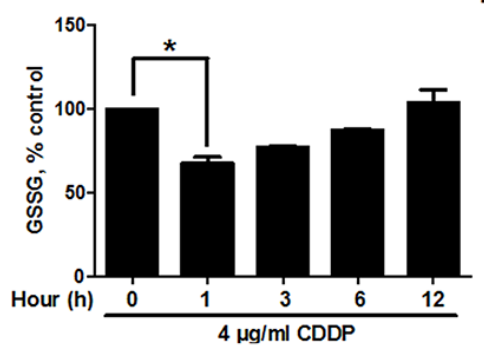

H

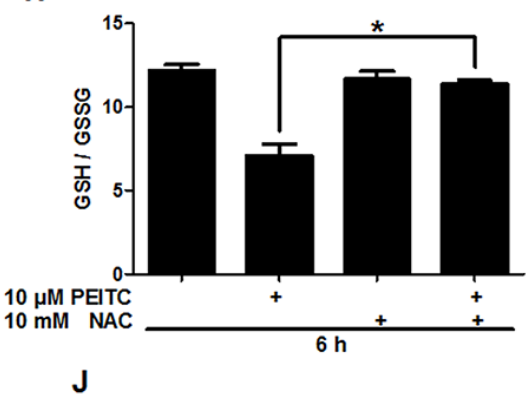

$$
\text { J }
$$

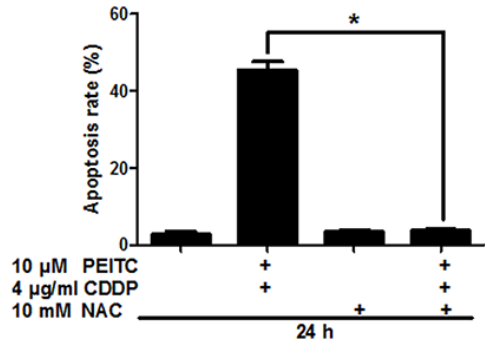

C
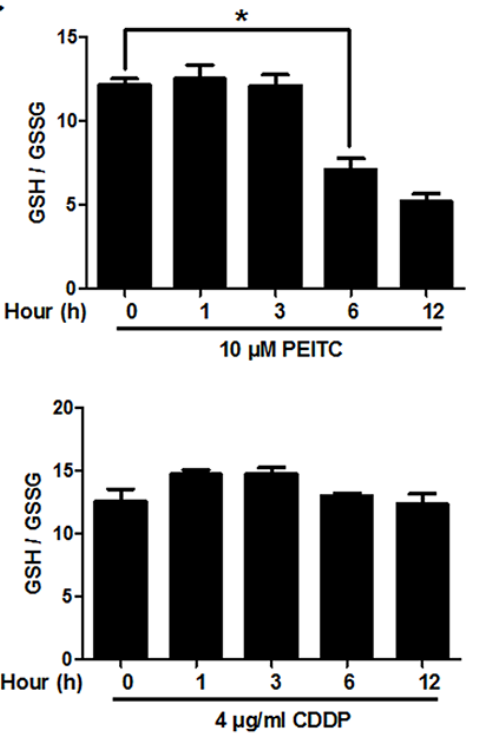

Figure 5: PEITC depletes GSH and decreases GSH/GSSG ratio. Analysis of (A) GSH levels, (B) GSSG levels and (C) GSH/ GSSG ratio in GBC-SD cells, after treatment with 10 uM PEITC for the indicated times. Analysis of (D) GSH levels, (E) GSSG levels and (F) GSH/GSSG ratio in GBC-SD cells treated with $4 \mathrm{ug} / \mathrm{ml}$ CDDP for the indicated times. (G) Effect of NAC on PEITC-induced GSH depletion. GBC-SD cells were preincubated with $10 \mathrm{mM} \mathrm{NAC}$ for $12 \mathrm{hrs}$ before treatment with 10 uM PEITC for 1 hour. (H) Effect of NAC on PEITC-induced decrease in GSH/GSSG ratio. GBC-SD cells were preincubated with $10 \mathrm{mM}$ NAC for 12 hrs before treatment with $10 \mathrm{uM}$ PEITC for $6 \mathrm{hrs}$. (I) Effect of NAC on PEITC-induced Mcl-1 degradation. GBC-SD cells were preincubated with $10 \mathrm{mM}$ NAC for $12 \mathrm{hrs}$ before treatment with $10 \mathrm{uM}$ PEITC for $18 \mathrm{hrs}$. Mcl-1 protein was detected by western blot and B-Actin was used as a loading control. (J) Effect of NAC on PEITC-CDDP-induced cell apoptosis. GBC-SD cells were preincubated with $10 \mathrm{mM}$ NAC for 12 hrs before treatment with PEITC-CDDP combination for $24 \mathrm{hrs}$. Cell apoptosis was detected by Annexin V/PI assays. Data shown is average of three independent experiments. $* P<0.05$. 
Despite the confirmed importance of Mcl-1 in several cancers, the role of Mcl-1 in BTC survival has yet to be explored. In this study, we provided evidence that the effectiveness of CDDP is partially dependent on the Mcl-1 expression. Previously we found that Mcl-1 expression is increased in gallbladder carcinoma tissues [28]. Therefore investigation into new therapeutic strategies targeting Mcl-1 could prove crucial in treating BTC.

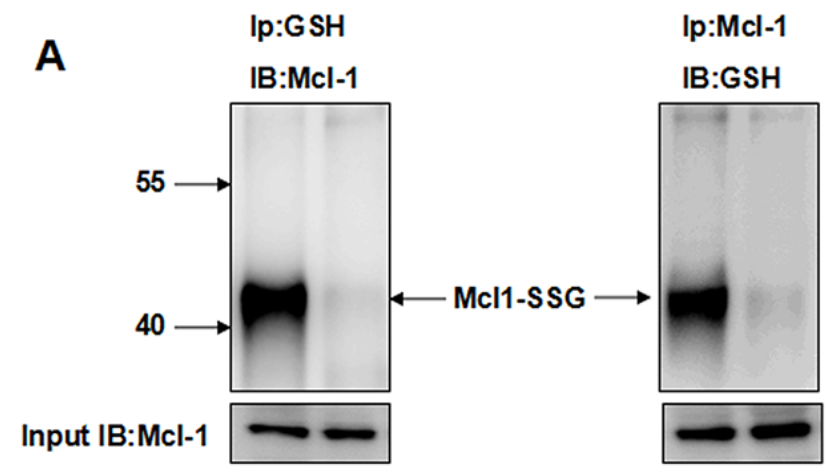

(Antibody) GSH IgG

(Antibody) Mcl-1 IgG

C

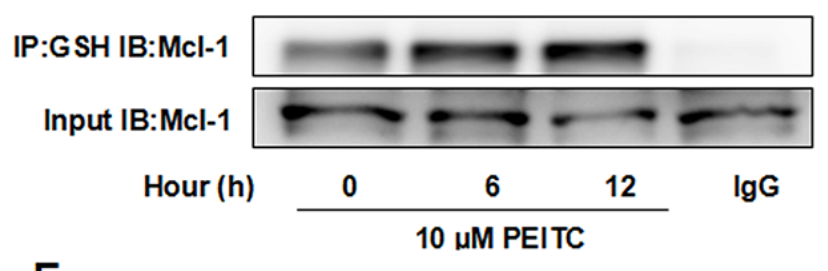

E
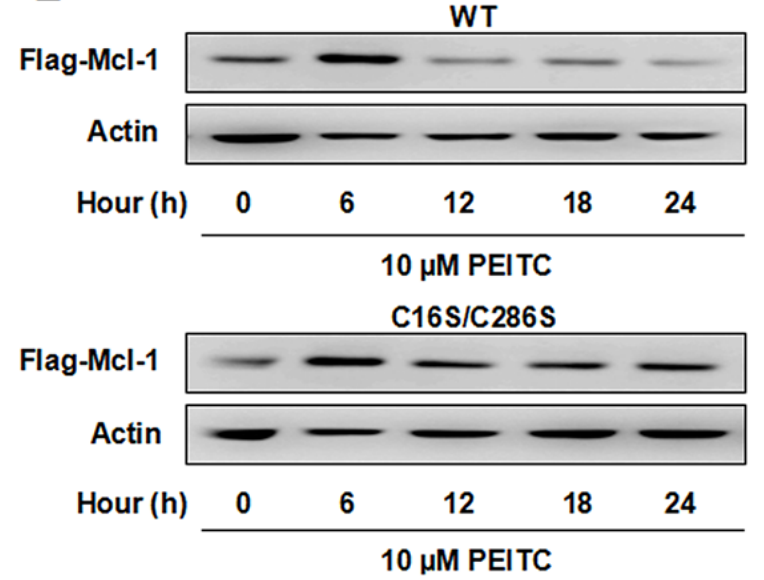

Several studies have discovered that PEITC is able to enhance the cytotoxic effect of CDDP in cancer cells $[10,11]$. But the synergistic effect of PEITC and CDDP in BTC cells remains unknown. Moreover, little information is available concerning the functional role of Mcl-1 in mediating PEITC-induced chemosensitization. In this study, we found that PEITC can significantly enhance CDDP-induced apoptosis by degrading Mcl-1 in BTC
B

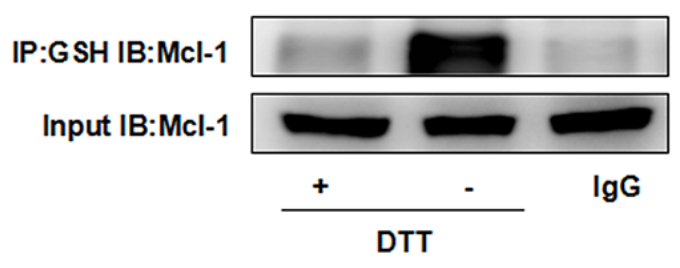

D

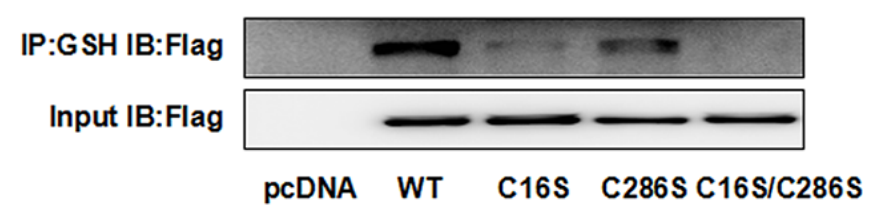

$\mathbf{F}$

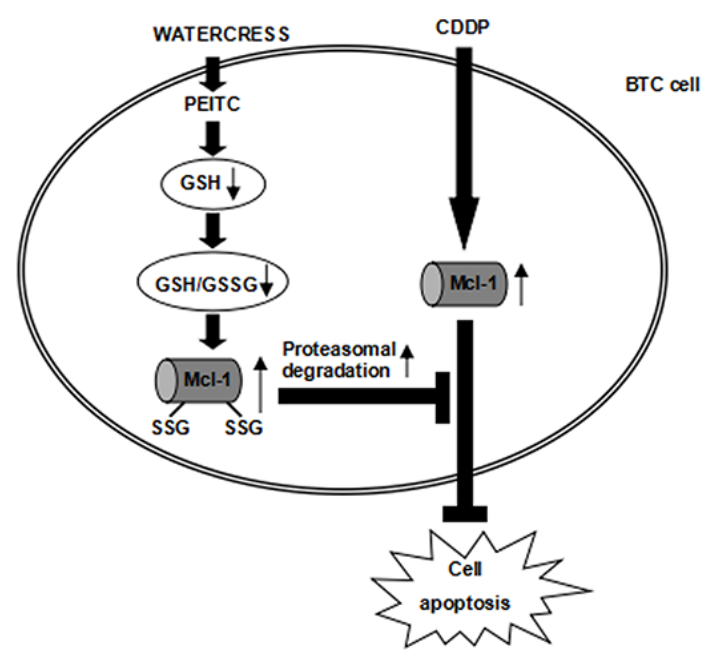

Figure 6: PEITC increases the glutathionylated Mcl-1 and induces glutathionylation-dependent degradation of Mcl-1. (A) Reciprocal IP/western analysis. Extracts from GBC-SD cells were immunoprecipitated using antibodies specific for Mcl-1 or GSH and the co-eluted proteins were detected by western blot with antibodies specific to GSH or Mcl-1. (B) GBC-SD cell lysate was treated with $10 \mathrm{mM}$ DTT for 10 min before IP and the level of glutathionylated Mcl-1 was detected. (C) GBC-SD cells were treated with 10 uM PEITC and harvested at the indicated times and the level of glutathionylated Mcl-1 was detected by IP. (D) After transfection with Flag-Mcl-1 WT, C16S, C286S or C16S/C286S mutant for 48 hrs, level of glutathionylated Flag-Mcl-1 was detected by IP. (E) After transfection with Flag-Mcl-1 WT or C16S/C286S mutant for 48 hrs, GBC-SD cells were treated with $10 \mathrm{uM}$ PEITC and harvested at the indicated times. Flag-Mcl-1 expression was detected by western blot and B-Actin was used as a loading control. (F) Proposed model of combination effect of PEITC and CDDP in BTC cells. CDDP can increase Mcl-1 protein level, which is partially responsible for CDDP resistance. PEITC can deplete GSH, decrease GSH/GSSG ratio and increase the glutathionylated Mcl-1, making Mcl-1 more susceptible to proteasomal degradation. Mcl1-SSG: the glutathionylated Mcl-1. IB: immunoblot. 
cells. Under mild oxidative stress, protein glutathionylation regulates functions of multiple proteins [18, 19]. Here, our results clearly show that Mcl-1 is glutathionylated in GBC-SD cells and a sub-toxic concentration of PEITC increases the glutathionylated $\mathrm{Mcl}-1$, followed by rapid proteasomal degradation. Whereas, Trachootham et al. showed that PEITC renders Mcl-1 more susceptible to cleavage by caspase-3 by de-glutathionylation of Mcl-1 in chronic lymphocytic leukemia cells [7]. We speculate that the discrepancy is due to different cell lines and different concentration of PEITC in two studies. PEITC may have different effect on pathways existing in different cells, and may impact degradation pathway of Mcl-1. By mutagenesis, we identified and confirmed that both Cys16 and Cys286 are glutathionylation sites on human Mcl-1 protein. Although there was a consistent correlation between glutathionylation levels and proteasomal degradation of Mcl-1, the double mutant could not be glutathionylated (Figure 6D) and only partially prevented proteasomal degradation (Figure 6E). This suggests that PEITC-mediated degradation of Mcl-1 may involve not only glutathionylation-dependent degradation but also other mechanisms. It would be interesting to examine other mechanisms of PEITC-mediated degradation of Mcl-1.

Conventional chemotherapies for cancer cells are believed to mainly eliminate the majority of differentiated cancer cells but spare cancer stem cells, which are thought to be associated with recurrence [29-31]. In the present study, we used SP cells from GBC-SD cells as a model of cancer stem-like cells and found that SP cells have higher Mcl-1 expression, consistent with a recent report [32]. Moreover, PEITC enhanced the efficacy of CDDP by degrading Mcl-1 in SP cells, which implies that PETIC is a promising chemotherapy-sensitizing agent targeting cancer stem cells.

Previous studies showed that eating watercress can significantly increase the blood level of PEITC in humans [9] and an oral dose of $40 \mathrm{mg}$ PEITC can result in a plasma concentration in the micromolar range within 3 to $8 \mathrm{hrs}$ [33]. Thus, the effective concentration of PEITC appears to be achievable by oral supplementation in humans. Furthermore, a Phase I trial showed that at lower doses (40 and $80 \mathrm{mg}$ daily for 30 days) PEITC was well-tolerated, and patients that consumed high doses of PEITC (120 and $160 \mathrm{mg}$ daily for 30 days) showed only minor toxicity with low-grade diarrhea [5]. The fact that PEITC is found in our normal diets combined with its relatively low toxicity in humans provides strong support for a clinical investigation of PEITC-CDDP co-treatment in BTC.

In conclusion, CDDP resistance is partially associated with Mcl-1 in BTC cells and PEITC can enhance CDDP-induced apoptosis via glutathionylationdependent degradation of $\mathrm{Mcl}-1$. These results not only identify a novel mechanism of PEITC-enhanced chemosensitivity of BTC cells to CDDP, but also provide support that dietary intake of watercress may help reverse CDDP resistance in BTC patients.

\section{MATERIALS AND METHODS}

\section{Cell culture and reagents}

The human gallbladder cancer (GBC-SD) and human cholangiocarcinoma (RBE) cell lines were obtained from Cell Bank, Shanghai Institutes for Biological Sciences, Chinese Academy of Sciences. GBC-SD and RBE cells were maintained in RPMI 1640 (Hyclone) supplemented with 10\% fetal bovine serum (Hyclone). Cells were cultured in a humidified atmosphere of 5\% $\mathrm{CO}_{2}$ at $37^{\circ} \mathrm{C}$.

PEITC, NAC, Hoechst 33342, verapamil, DTT and cycloheximide were purchased from Sigma. MG132 was purchased from Calbiochem and CDDP was obtained from Qilu Pharmaceutical Co., Ltd (Jinan, China). PEITC was dissolved in dimethyl sulfoxide (DMSO) and was freshly diluted in culture media before use in experiments.

\section{SP cell sorting from GBC-SD cells}

SP analysis was performed as previously described [34]. Briefly, cells were trypsinized and resuspended in icecold Hank's balanced salt solution (Invitrogen). Hoechst 33342 was added at a final concentration of $5 \mathrm{mg} / \mathrm{ml}$ in the presence or absence of $50 \mathrm{mg} / \mathrm{ml}$ verapami. After incubating at $37^{\circ} \mathrm{C}$ for $90 \mathrm{~min}$, the cells were analyzed by flow cytometry.

\section{Cell viability, apoptosis analysis, CI and DRI}

Cell viability was assayed using the MTT assay (Sigma) as previously described [35]. Cell apoptosis was assessed using an Annexin V-FITC/ PI kit (BD Pharmingen) and analyzed by flow cytometry on FACS Calibur (Becton Dickson) [36]. Cells that were positively stained by Annexin V-FITC only (early apoptosis) and positive for both Annexin V-FITC and PI (late apoptosis) were quantitated and both subpopulations were considered as overall apoptotic cells.

CI and DRI values of PEITC-CDDP co-treatment were determined using the CalcuSyn software using nonconstant ratio combination design as previously described $[37,38]$.

\section{Reverse transcription and real-time PCR}

Reverse transcription and quantitative real-time PCR were carried out as previously described [39]. Primers sequences used in PCR analysis were as follows: Mcl-1 forward 5'-TCCAAGGCATGCTTCGGA-3' and reverse 5'-GGCACCAAAAGAAATGAGAGTCAC-3'; GAPDH forward 5'-GAAGGTGAAGGTCGGAGTC-3' and reverse 5'-GAAGATGGTGATGGGATTTC-3'. Human GADPH mRNA served as an endogenous control for normalization. 


\section{GSH/GSSG ratio assay}

GSH is a tripeptide with a free thiol group and function as a major antioxidant in cells. GSH/GSSG ratio reflects the cellular redox state. The total GSH and GSSG were determined by colorimetric microplate assay kit (Beyotime, China) as previously described [40, 41]. GSH/ GSSG ratio was obtained $\{$ ratio $=($ Total GSH $-2 \mathrm{GSSG}) /$ GSSG\}.

\section{Mcl-1 siRNA and mutant transfection}

Two specific siRNA oligonucleotides complementary to the $M c l-1$ mRNA sequence were transiently transfected into GBC-SD cells using Lipofectamine 2000 (Invitrogen) as previously described [42, 43]. A non-specific siRNA was also transfected as a mock control. After $48 \mathrm{hrs,} \mathrm{GBC-SD}$ cells were either lysed for western blot analysis or were exposed to CDDP for an additional $24 \mathrm{hrs}$ and analysed for apoptosis using the methods described above. The siRNA-1 sequences complimentary for $M c 1-1$ mRNA were $5^{\prime}$ GUAUCACAGACGUUCUCGUdTdT 3' and 3' dTdTCAU AGUGUCUGCAAGAGCA 5'. The siRNA-2 sequences complimentary for $\mathrm{Mcl}-1$ mRNA were 5' GGACUUUU AGAUUUAGUGAdTdT 3' and 3' dTdTCCUGAAAAUC UAAAUCACU $5^{\prime}$.

Site-directed mutagenesis of the $\mathrm{Mcl}-1$ gene was performed using the Quick Change Lightning SiteDirected Mutagenesis Kit (Agilent Technologies) using the pcDNA3.1-Flag-Mcl-1 plasmid as the template. Cells were transiently transfected with Flag-Mcl-1 WT and mutants using the above-mentioned Lipofectamine method. Nonrelevant plasmid pcDNA3.1 was used as the transfection control. After $48 \mathrm{hrs,} \mathrm{GBC-SD} \mathrm{cells} \mathrm{were}$ lysed for immunoprecipitation (IP) or treated with PEITC for an additional $24 \mathrm{hrs}$ and analysed by western blot. The anti-Flag monoclonal antibody was purchased from Sigma.

\section{Assays of Mcl-1 expression and glutathionylation}

Mcl-1, Bcl-xl and Bcl-2 protein levels were determined by western blot as previously described [44]. Cellular glutathionylation of Mcl-1 was determined by IP with an anti-GSH antibody under non-reducing conditions, followed by western blot analysis $[7,18]$ using an anti-Mcl-1 antibody. Antibodies to Bcl-2 and Bcl-xl were purchased from Santa Cruz. Antibodies to GSH and Mcl-1 were purchased from Abcam.

\section{In vivo study in tumor-bearing mice}

All animal experiments were done in accordance with institutional guidelines for animal welfare. GBC-SD cells were harvested, washed, and resuspended in serum-free RPMI 1640 and then injected subcutaneously into 6-week old BALB/c-nu/nu mice ( $n=28$ mice, purchased from Shanghai Experimental Animal Center). Tumor volumes were measured twice a week using a calliper, and were calculated using the formula $\mathrm{V}=\pi / 6 \times$ length $\times$ width $^{2}$ $[34,45]$. When the tumor size was approximately $50 \mathrm{~mm}^{3}$, the mice were sorted into four equal groups $(n=7$ mice per group). The tumor-bearing mice were intra-peritoneally administered with physiological saline as a control, PEITC $(25 \mathrm{mg} / \mathrm{kg})$, CDDP $(2.5 \mathrm{mg} / \mathrm{kg})$ or PEITC/CDDP twice a week. Tumor volumes were measured twice a week with a calliper and body weights were also recorded. The mice were sacrificed after 10 days, and body weight and tumor weight were measured.

\section{Statistical analysis}

Data were shown as mean value \pm SD. SPSS17.0 software was used for statistical analysis. Analysis of variance was applied for comparison of the means of two or multiple groups. A value of $P<0.05$ was considered significant.

\section{ACKNOWLEDGMENTS}

We thank Prof. R. David Andrew for sharing the Mcl-1 plasmid.

\section{GRANT SUPPORT}

This work was supported by grants from National Natural Science Foundation of China (81072011, J. Wang), National Key Technology R \& D Program (2012BAI06B01, J. Wang), Shanghai Science and Technology Fund (12XD1403400, J. Wang) and Shanghai Municipal Public Health Bureau, China (XBR2011035, J. Wang).

\section{CONFLICTS OF INTEREST}

No potential conflicts of interest were disclosed.

\section{REFERENCES}

1. de Groen PC, Gores GJ, LaRusso NF, Gunderson LL, Nagorney DM. Biliary tract cancers. N Engl J Med. 1999; 341:1368-1378.

2. Valle JW, Furuse J, Jitlal M, Beare S, Mizuno N, Wasan H, Bridgewater J, Okusaka T. Cisplatin and gemcitabine for advanced biliary tract cancer: a meta-analysis of two randomised trials. Ann Oncol. 2014; 25:391-398.

3. Matsuda $T$, Marugame $T$. International comparisons of cumulative risk of gallbladder cancer and other biliary tract cancer, from Cancer Incidence in Five Continents Vol. VIII. Jpn J Clin Oncol. 2007; 37:74-75.

4. Valle J, Wasan H, Palmer DH, Cunningham D, Anthoney A, Maraveyas A, Madhusudan S, Iveson T, Hughes S, Pereira $\mathrm{SP}$, Roughton $\mathrm{M}$, Bridgewater $\mathrm{J}$, Investigators $\mathrm{ABCT}$. 
Cisplatin plus gemcitabine versus gemcitabine for biliary tract cancer. N Engl J Med. 2010; 362:1273-1281.

5. Wang X, Govind S, Sajankila SP, Mi L, Roy R, Chung FL. Phenethyl isothiocyanate sensitizes human cervical cancer cells to apoptosis induced by cisplatin. Mol Nutr Food Res. 2011; 55:1572-1581.

6. Trachootham D, Zhou Y, Zhang H, Demizu Y, Chen Z, Pelicano H, Chiao PJ, Achanta G, Arlinghaus RB, Liu J, Huang P. Selective killing of oncogenically transformed cells through a ROS-mediated mechanism by betaphenylethyl isothiocyanate. Cancer Cell. 2006; 10:241-252.

7. Trachootham D, Zhang H, Zhang W, Feng L, Du M, Zhou Y, Chen Z, Pelicano H, Plunkett W, Wierda WG, Keating MJ, Huang P. Effective elimination of fludarabine-resistant CLL cells by PEITC through a redox-mediated mechanism. Blood. 2008; 112:1912-1922.

8. Xiao D, Powolny AA, Moura MB, Kelley EE, Bommareddy A, Kim SH, Hahm ER, Normolle D, Van Houten B, Singh SV. Phenethyl isothiocyanate inhibits oxidative phosphorylation to trigger reactive oxygen species-mediated death of human prostate cancer cells. J Biol Chem. 2010; 285:26558-26569.

9. Syed Alwi SS, Cavell BE, Telang U, Morris ME, Parry BM, Packham $\mathrm{G}$. In vivo modulation of $4 \mathrm{E}$ binding protein 1 (4E-BP1) phosphorylation by watercress: a pilot study. Br J Nutr. 2010; 104:1288-1296.

10. Denis I, Cellerin L, Gregoire M, Blanquart C. Cisplatin in combination with Phenethyl Isothiocyanate (PEITC), a potential new therapeutic strategy for malignant pleural mesothelioma. Oncotarget. 2014; 5:11641-11652. doi: 10.18632/oncotarget.2604.

11. Wu WJ, Zhang Y, Zeng ZL, Li XB, Hu KS, Luo HY, Yang J, Huang $\mathrm{P}, \mathrm{Xu} \mathrm{RH}$. beta-phenylethyl isothiocyanate reverses platinum resistance by a GSH-dependent mechanism in cancer cells with epithelial-mesenchymal transition phenotype. Biochem Pharmacol. 2013; 85:486-496.

12. Di Pasqua AJ, Hong C, Wu MY, McCracken E, Wang X, Mi L and Chung FL. Sensitization of non-small cell lung cancer cells to cisplatin by naturally occurring isothiocyanates. Chem Res Toxicol. 2010; 23:1307-1309.

13. Oka M, Toyoda C, Kaneko Y, Nakazawa Y, Aizu-Yokota E, Takehana M. Characterization, localization of side population cells in the lens. Mol Vis. 2010; 16:945-953.

14. Tabor MH, Clay MR, Owen JH, Bradford CR, Carey TE, Wolf GT, Prince ME. Head, neck cancer stem cells: the side population. Laryngoscope. 2011; 121:527-533.

15. Wan G, Zhou L, Xie M, Chen H, Tian J. Characterization of side population cells from laryngeal cancer cell lines. Head Neck. 2010; 32:1302-1309.

16. Wu C, Alman BA. Side population cells in human cancers. Cancer Lett. 2008; 268:1-9.

17. Chen HH, Song IS, Hossain A, Choi MK, Yamane Y, Liang ZD, Lu J, Wu LY, Siddik ZH, Klomp LW, Savaraj N, Kuo MT. Elevated glutathione levels confer cellular sensitization to cisplatin toxicity by up-regulation of copper transporter hCtr1. Mol Pharmacol. 2008; 74:697-704.

18. Velu CS, Niture SK, Doneanu CE, Pattabiraman N, Srivenugopal KS. Human p53 is inhibited by glutathionylation of cysteines present in the proximal DNAbinding domain during oxidative stress. Biochemistry. 2007; 46:7765-7780.

19. Ghezzi P. Regulation of protein function by glutathionylation. Free Radic Res. 2005; 39:573-580.

20. Hung CC, Chien CY, Chiang WF, Lin CS, Hour TC, Chen HR, Wang LF, Ko JY, Chang CH, Chen JY. p22phox confers resistance to cisplatin, by blocking its entry into the nucleus. Oncotarget. 2015; 6:4110-4125. doi: 10.18632/oncotarget.2893.

21. Garcia-Cano J, Ambroise G, Pascual-Serra R, Carrion MC, Serrano-Oviedo L, Ortega-Muelas M, Cimas FJ, Sabater S, Ruiz-Hidalgo MJ, Sanchez Perez I, Mas A, Jalon FA, Vazquez A, et al. Exploiting the potential of autophagy in cisplatin therapy: A new strategy to overcome resistance. Oncotarget. 2015; 6:15551-15565. doi: 10.18632/ oncotarget.3902.

22. Muller CB, De Bastiani MA, Becker M, Franca FS, Branco MA, Castro MA, Klamt F. Potential crosstalk between cofilin-1 and EGFR pathways in cisplatin resistance of nonsmall-cell lung cancer. Oncotarget. 2015; 6:3531-3539. doi: 10.18632/oncotarget.3471.

23. Su WP, Hsu SH, Wu CK, Chang SB, Lin YJ, Yang WB, Hung JJ, Chiu WT, Tzeng SF, Tseng YL, Chang JY, Su WC, Liaw H. Chronic treatment with cisplatin induces replicationdependent sister chromatid recombination to confer cisplatinresistant phenotype in nasopharyngeal carcinoma. Oncotarget. 2014; 5:6323-6337. doi: 10.18632/oncotarget.2210.

24. Wang Q, Shi S, He W, Padilla MT, Zhang L, Wang X, Zhang B, Lin Y. Retaining MKP1 expression and attenuating JNKmediated apoptosis by RIP1 for cisplatin resistance through miR-940 inhibition. Oncotarget. 2014; 5:1304-1314. doi: 10.18632/oncotarget.1798.

25. Sorensen BH, Thorsteinsdottir UA, Lambert IH. Acquired Cisplatin Resistance in Humane Ovarian cancer A2780 cells correlates with shift in Taurine homeostasis and ability to volume regulate. Am J Physiol Cell Physiol. 2014; 307:C1071-80. doi: 10.1152/ajpcell.00274.2014.

26. Heiser D, Labi V, Erlacher M, Villunger A. The Bcl-2 protein family and its role in the development of neoplastic disease. Exp Gerontol. 2004; 39:1125-1135.

27. Maji S, Samal SK, Pattanaik L, Panda S, Quinn BA, Das SK, Sarkar D, Pellecchia M, Fisher PB, Dash R. Mcl-1 is an important therapeutic target for oral squamous cell carcinomas. Oncotarget. 2015; 6:16623-37. doi: 10.18632/oncotarget.3932.

28. Wang W, Yin X, Li G, Yi J, Wang J. Expressions of farnesoid $\mathrm{X}$ receptor and myeloid cell leukemia sequence 1 protein are associated with poor prognosis in patients with gallbladder cancer. Chin Med J (Engl). 2014; 127:2637-2642.

29. Davies AH, Reipas K, Hu K, Berns R, Firmino N, Stratford AL, Dunn SE. Inhibition of RSK with the novel small-molecule 
inhibitor LJI308 overcomes chemoresistance by eliminating cancer stem cells. Oncotarget. 2015; 6:20570-7. doi: 10.18632/oncotarget.4135.

30. Dean M, Fojo T, Bates S. Tumour stem cells and drug resistance. Nat Rev Cancer. 2005; 5:275-284.

31. Donnenberg VS, Donnenberg AD. Multiple drug resistance in cancer revisited: the cancer stem cell hypothesis. J Clin Pharmacol. 2005; 45:872-877.

32. Singh S, Bora-Singhal N, Kroeger J, Laklai H, Chellappan SP. betaArrestin-1 and Mcl-1 modulate selfrenewal growth of cancer stem-like side-population cells in non-small cell lung cancer. PLoS One. 2013; 8:e55982.

33. Liebes L, Conaway CC, Hochster H, Mendoza S, Hecht SS, Crowell J, Chung FL. High-performance liquid chromatography-based determination of total isothiocyanate levels in human plasma: application to studies with 2-phenethyl isothiocyanate. Anal Biochem. 2001; 291:279 289.

34. Li XX, Dong Y, Wang W, Wang HL, Chen YY, Shi GY, Yi J, Wang J. Emodin as an effective agent in targeting cancer stem-like side population cells of gallbladder carcinoma. Stem Cells Dev. 2013; 22:554-566.

35. Yi J, Yang J, He R, Gao F, Sang H, Tang X, Ye RD. Emodin enhances arsenic trioxide-induced apoptosis via generation of reactive oxygen species and inhibition of survival signaling. Cancer Res. 2004; 64:108-116.

36. Jing Y, Yang J, Wang Y, Li H, Chen Y, Hu Q, Shi G, Tang X, Yi J. Alteration of subcellular redox equilibrium and the consequent oxidative modification of nuclear factor kappaB are critical for anticancer cytotoxicity by emodin, a reactive oxygen species-producing agent. Free Radic Biol Med. 2006; 40:2183-2197.

37. Han T, Fernandez M, Chou TC, Agarwal RP. 2-Chloro-2'deoxyadenosine synergistically enhances azidothymidine cytotoxicity in azidothymidine resistant T-lymphoid cells. Biochem Biophys Res Commun. 2004; 316:518-522.

38. Yaacob NS, Kamal NN, Norazmi MN. Synergistic anticancer effects of a bioactive subfraction of Strobilanthes crispus, tamoxifen on MCF-7 and MDA-MB-231 human breast cancer cell lines. BMC Complement Altern Med. 2014; 14:252.
39. Huang C, Han Y, Wang Y, Sun X, Yan S, Yeh ET, Chen Y, Cang H, Li H, Shi G, Cheng J, Tang X, Yi J. SENP3 is responsible for HIF-1 transactivation under mild oxidative stress via p300 de-SUMOylation. EMBO J. 2009; 28:2748-2762.

40. Rahman I, Kode A, Biswas SK. Assay for quantitative determination of glutathione and glutathione disulfide levels using enzymatic recycling method. Nat Protoc. 2006; 1:3159-3165.

41. Fan J, Cai H, Yang S, Yan L, Tan W. Comparison between the effects of normoxia and hypoxia on antioxidant enzymes and glutathione redox state in ex vivo culture of CD34(+) cells. Comp Biochem Physiol B Biochem Mol Biol. 2008; 151:153-158.

42. Wang W, Sun YP, Huang XZ, He M, Chen YY, Shi GY, Li H, Yi J, Wang J. Emodin enhances sensitivity of gallbladder cancer cells to platinum drugs via glutathion depletion and MRP1 downregulation. Biochem Pharmacol. 2010; 79:1134-1140.

43. Wang H, Li X, Chen T, Wang W, Liu Q, Li H, Yi J, Wang J. Mechanisms of verapamil-enhanced chemosensitivity of gallbladder cancer cells to platinum drugs: glutathione reduction and MRP1 downregulation. Oncol Rep. 2013; 29:676-684.

44. Han Y, Huang C, Sun X, Xiang B, Wang M, Yeh ET, Chen Y, Li H, Shi G, Cang H, Sun Y, Wang J, Wang W, et al. SENP3-mediated de-conjugation of SUMO2/3 from promyelocytic leukemia is correlated with accelerated cell proliferation under mild oxidative stress. J Biol Chem. 2010; 285:12906-12915.

45. Cha TL, Qiu L, Chen CT, Wen Y, Hung MC. Emodin downregulates androgen receptor and inhibits prostate cancer cell growth. Cancer Res. 2005; 65:2287-2295. 